\title{
Synthesis of ionic liquid-supported Schiff bases
}

\author{
Bin Li, Yi-Qun Li,* Wen-Jie Zheng, and Mei-Yun Zhou \\ Department of Chemistry, Jinan University, Guangzhou, 510632, China \\ E-mail: tlyq@jnu.edu.cn
}

\begin{abstract}
Some ionic liquid-supported Schiff bases 3 have been synthesized by condensation of aromatic aldehydes 2 with the ionic liquid 1-(2-aminoethyl)-3-methylimidazolium hexafluorophosphate 1 without solvent. The structure of 3a has been proven by single crystal X-ray crystallography.
\end{abstract}

Keywords: Ionic liquid, Schiff base, synthesis, X-ray crystal structure

\section{Introduction}

In recent years, ionic liquids have received considerable attention as an attractive alternative to the traditional organic solvents. ${ }^{1-4}$ The increased interest for their investigation is mainly due to their unique properties, such as chemical and thermal stability, high polarity, negligible vapor pressure, high electrical conductivity. ${ }^{5-8}$ In addition, the miscibility of ionic liquids with organic solvents or water can be tuned by the choice of cations and anions. Owing to these properties, research has been focused on the synthesis of new ionic liquids with functional groups, so called "task-specific" or functionalized ionic liquids, and their applications. ${ }^{9-11}$

Schiff bases are usually formed by the condensation of a primary amine (or diamine) with an aldehyde. ${ }^{12-14}$ Numerous reports on the preparation of Schiff bases and their applications have been published. Schiff bases are one of most prevalent mixed-donor ligands in coordination chemistry. ${ }^{15}$ In addition, Schiff bases and their complexes are widely applied in biochemistry, material science, catalysis, encapsulation, activation, transport and separation phenomena, hydrometallurgy. ${ }^{16-19}$

As part of our program aimed at developing a novel functionalized ionic liquid, we have synthesized a series of ionic liquid-supported Schiff bases. 


\section{Results and Discussion}

\section{Chemistry}

Ionic liquid-supported Schiff bases 3a-i were synthesized by condensation of the ionic liquid 1(2-aminoethyl)-3-methylimidazolium hexafluorophosphate (1) with aromatic aldehydes 2a-i at room temperature without both catalyst and solvent (Scheme 1).

A clear phase change was observed in all of the reactive systems investigated. The slurry of reactants eventually solidified after completion of the reaction forming the solid product.

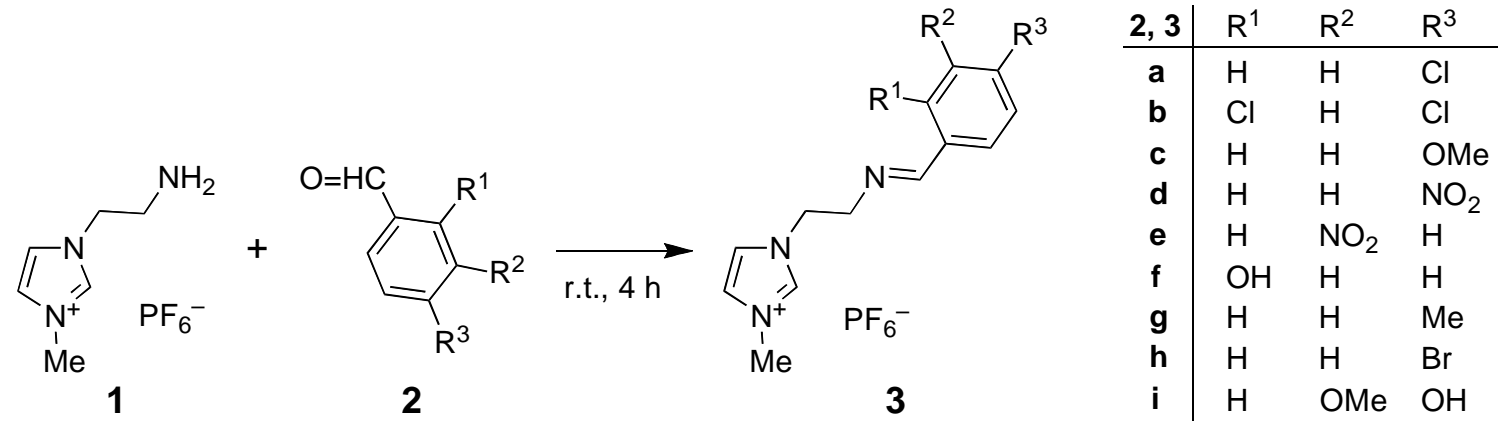

\section{Scheme 1}

The reaction yields (between 21\% and 54\%) are low and/or moderate, which may be due to the reversible process of the condensation reaction. It should be mentioned that the nature of various substituents at the aromatic aldehydes has no obvious effect on yields.

The structure of products was assigned on the basis of spectroscopic data. In IR spectra, compounds 3 exhibit absorption bands around $1640 \mathrm{~cm}^{-1}$ for the $\mathrm{C}=\mathrm{N}$ group. The stretching vibration of $\mathrm{H}-\mathrm{C}(=\mathrm{N})$ appears in the region between 3100 and $3200 \mathrm{~cm}^{-1}$. In ${ }^{1} \mathrm{H}$ NMR spectra, the chemical shift of $\mathrm{HC}=\mathrm{N}$ is displayed at $\delta 7.30-7.80$; an exception is compound $3 \mathrm{f}$ with $\delta 8.50$ presumably due to the effect of the ortho-hydroxyl group in the aromatic ring.

\section{X-ray crystallography}

An X-ray structure drawing of compound 3a is shown in Figure 1. The crystal data structure refinement for compound 3a is summarized in Table 1.

The single-crystal structure analysis reveals that complex contains one cation $\left(\mathrm{C}_{13} \mathrm{H}_{15} \mathrm{ClN}_{3}\right)^{+}$ and one $\mathrm{PF}_{6}{ }^{-}$anion. The imidazole ring $[\mathrm{N}(2), \mathrm{C}(10), \mathrm{N}(3), \mathrm{C}(11), \mathrm{C}(12)]$ and aromatic ring $[\mathrm{C}(1), \mathrm{C}(2), \mathrm{C}(3), \mathrm{C}(4), \mathrm{C}(5), \mathrm{C}(6)]$ are not coplanar with a dihedral angle of $52.84^{\circ}$ between them. As expected, the molecular structure adopts a trans configuration about the $\mathrm{C}(7)=\mathrm{N}(1)$ double bond. Within the imidazole fragments, the $\mathrm{N}(2)-\mathrm{C}(10)$ and $\mathrm{N}(3)-\mathrm{C}(10)$ are $1.328(2)$ and 1.323(2) $\AA$, respectively, which is remarkably shorter than the normal $\mathrm{C}-\mathrm{N}$ bond $(1.47 \AA)$ but longer than the typical $\mathrm{C}=\mathrm{N}$ bond $(1.28 \AA) .{ }^{20}$ The $\mathrm{C}(11)-\mathrm{C}(12)$ bond length is $1.345(3) \AA$, remarkably shorter than the typical $\mathrm{C}-\mathrm{C}$ single bond $(1.478 \AA)^{20}$ due to the aromatic conjugated 
system of the imidazole ring. In addition, as compared with normal $\mathrm{C}=\mathrm{N}$ double bond values in other Schiff bases, the shorter $\mathrm{C}(7)=\mathrm{N}(1)$ double bond distance of 1.272(3) $\AA$ may be attributable to the $\pi$-conjugated system of the aromatic ring and the $\mathrm{C}=\mathrm{N}$ bond, too.

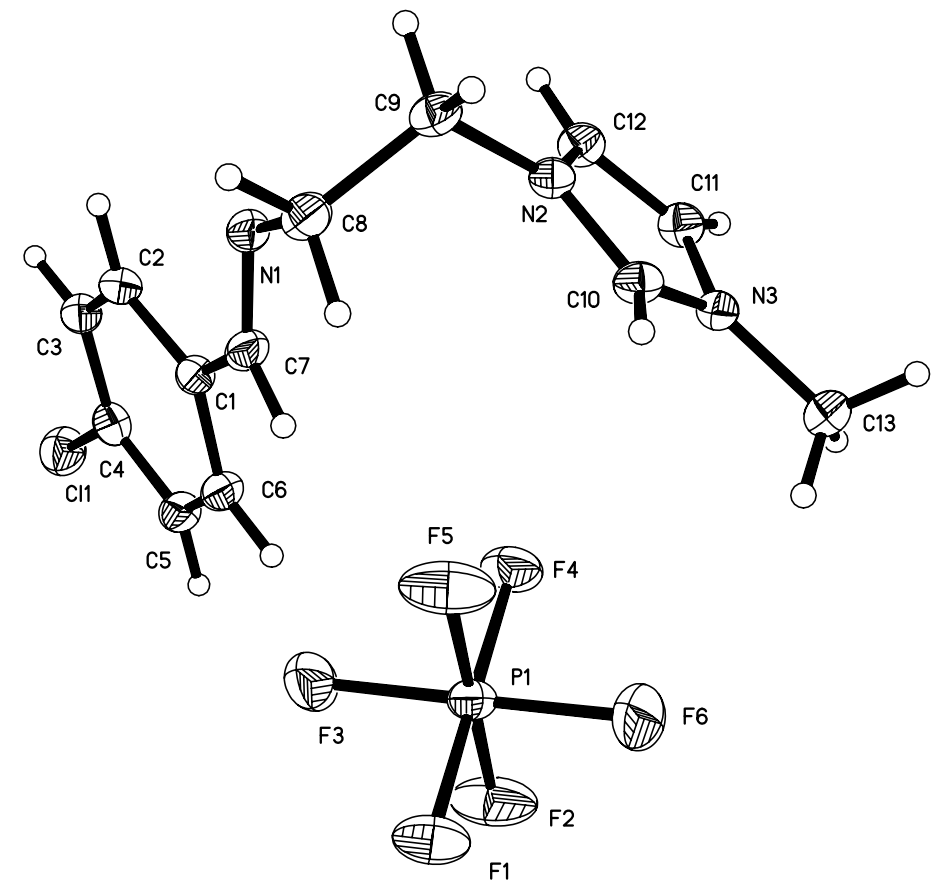

Figure 1. Crystal structure of compound 3a. 
Table 1. Crystal data structure refinement for compound 3a

\begin{tabular}{ll}
\hline Empirical formula & $\mathrm{C}_{13} \mathrm{H}_{15} \mathrm{ClF}_{6} \mathrm{~N}_{3} \mathrm{P}$ \\
Formula weight & 393.70 \\
Temperature & $173(2) \mathrm{K}$ \\
Wavelength & $0.71073 \AA$ \\
Crystal system, space group & Triclinic, $\mathrm{P}-1$ \\
Unit cell dimensions & $\mathrm{a}=8.2698(13) \AA \alpha=112.286(2)^{\circ}$ \\
& $\mathrm{b}=9.8891(16) \AA \beta=97.309(3)^{\circ}$ \\
& $\mathrm{c}=11.5980(19) \AA \gamma=105.511(2)^{\circ}$ \\
Volume & $817.4(2) \AA^{3}$ \\
Z, Calculated density & $2,1.600 \mathrm{Mg} / \mathrm{m}^{3}$ \\
Absorption coefficient & $0.395 \mathrm{~mm}^{-1}$ \\
F(000) & 400 \\
Crystal size & $0.45 \times 0.42 \times 0.21 \mathrm{~mm}$ \\
Theta range for data collection & 1.96 to $27.03^{\circ}$ \\
Limiting indices & $-10<=\mathrm{h}<=10,-12<=\mathrm{k}<=12,-14<=1<=14$ \\
Reflections collected/unique & $6879 / 3507[\mathrm{R}(\mathrm{int})=0.0182]$ \\
Completeness to theta $=27.03$ & $97.8 \%$ \\
Absorption correction & $\mathrm{Semi}-\mathrm{empirical}$ from equivalents \\
Max. and min. transmission & 0.9216 and 0.8423 \\
Refinement method & Full-matrix least-squares on $\mathrm{F}^{2}$ \\
Data / restraints / parameters & $3507 / 0 / 218$ \\
Goodness-of-fit on $\mathrm{F}^{2}$ & 1.079 \\
Final R indices [I $>$ 2sigma(I) $]$ & $\mathrm{R} 1=0.0380, \mathrm{wR} 2=0.1161$ \\
$\mathrm{R}$ indices (all data) & $\mathrm{R} 1=0.0488, \mathrm{wR} 2=0.1321$ \\
Largest diff. peak and hole & 0.405 and $-0.355 \mathrm{e} \cdot \AA^{-3}$ \\
\hline
\end{tabular}

\section{Experimental Section}

General Procedures. Melting points were measured on an Electrothemal X6 microscopy digital melting point apparatus. IR spectra were recorded on a Nicolet 6700 spectrometer using $\mathrm{KBr}$ pellets. ${ }^{1} \mathrm{H}$ NMR spectra were recorded in DMSO- $d_{6}$ on a Bruker AVANCE $300(300 \mathrm{MHz})$ instrument with the TMS as an internal standard. Elemental analyses were carried out on a PE EA2400 CHN analyzer. The ionic liquid was prepared according to the method reported. ${ }^{21}$

(E)-1-[2-(Arylideneamino)ethyl]-3-methyl-1H-imidazol-3-ium hexafluorophosphates (3). A mixture of 1-(2-aminoethyl)-3-methylimidazolium hexafluorophosphate (1, $1.028 \mathrm{~g}, 4 \mathrm{mmol})$ and aromatic aldehydes $(2,3 \mathrm{mmol})$ without solvent was stirred at room temperature for $4 \mathrm{~h}$. After completion of the reaction, ethanol $(30 \mathrm{~mL})$ was added to the reaction mixture, the solid 
product formed was filtered off and washed with cold ethanol. The crude product was purified by recrystallization from ethanol/ethyl acetate $(3: 1 \mathrm{v} / \mathrm{v})$.

(E)-3-[2-(4-Chlorobenzylideneamino)ethyl]-1-methyl-1H-imidazol-3-ium hexafluorophosphate (3a). White solid $(0.25 \mathrm{~g}, 21.17 \%)$; mp 103-105 ${ }^{\circ} \mathrm{C}$. IR $(\mathrm{KBr}), \mathrm{cm}^{-1}: 1641(\mathrm{C}=\mathrm{N}), 827(\mathrm{P}-$ F). ${ }^{1} \mathrm{H}$ NMR (300 MHz, DMSO-d $)$ ): $\delta 3.07\left(\mathrm{~s}, 3 \mathrm{H}, \mathrm{CH}_{3}\right), 3.18\left(\mathrm{t}, J=5.35 \mathrm{~Hz}, 2 \mathrm{H}, \mathrm{CH}_{2}\right), 3.73(\mathrm{t}$, $\left.J=5.40 \mathrm{~Hz}, 2 \mathrm{H}, \mathrm{CH}_{2}\right), 6.60,6.64\left(\mathrm{AA}^{\prime}, 2 \mathrm{H}, \mathrm{Ar}-\mathrm{H}\right), 6.70(\mathrm{~s}, 1 \mathrm{H}, \mathrm{NCH}), 6.80(\mathrm{~s}, 1 \mathrm{H}, \mathrm{NCH}), 6.89$, $6.92\left(\mathrm{BB}\right.$ ', 2H, Ar-H), $7.47(\mathrm{~s}, 1 \mathrm{H}, \mathrm{N}=\mathrm{CH})$. Anal. calcd. for $\mathrm{C}_{13} \mathrm{H}_{15} \mathrm{ClF}_{6} \mathrm{~N}_{3} \mathrm{P}: \mathrm{C}, 39.66 ; \mathrm{H}, 3.84$; N, 10.67. Found: C, 39.27; H, 3.51; N, 10.42.

X-ray single-crystal data for compound 3a were collected at 173(2) K on a Bruker SMART CCD diffractometer equipped with a graphite-monochromatic Mok $\alpha$ radiation $(\lambda=0.71073 \AA)$ using an $\omega$ scan technique. Crystal data absorption corrections were made with the SADABS program. $^{22}$ The structure was solved by direct methods and refined by full-matrix least-squares techniques using SHELSL-97. ${ }^{23}$ The non-hydrogen atoms were refined anisotropically, and hydrogen atoms were introduced geometrically.

Full crystallographic data for the structure has been deposited at the Cambridge Crystallographic Data Centre (CCDC reference number 726047). Copies of the data can be obtained free of charge on application to CCDC, 12 Union Road, Cambridge, CB2 1EZ, UK (e-mail: deposit@ccdc.cam.ac.uk; fax: +44 1223-336-033).

1-[2-(2,4-Dichlorobenzylideneamino)ethyl]-3-methyl-3H-imidazol-3-ium hexafluorophosphate (3b). White solid (0.53 g, 41.26\%); mp 129-131 ${ }^{\circ} \mathrm{C}$. IR (KBr): $1638(\mathrm{C}=\mathrm{N}), 835 \mathrm{~cm}^{-1}(\mathrm{P}-$ F). ${ }^{1} \mathrm{H}$ NMR (300 MHz, DMSO-d $)$ ): $\delta 3.08\left(\mathrm{~s}, 3 \mathrm{H}, \mathrm{CH}_{3}\right), 3.23\left(\mathrm{t}, J=5.43 \mathrm{~Hz}, 2 \mathrm{H}, \mathrm{CH}_{2}\right), 3.75(\mathrm{t}$, $\left.J=5.43 \mathrm{~Hz}, 2 \mathrm{H}, \mathrm{CH}_{2}\right), 6.58(\mathrm{~d}, J=8.61 \mathrm{~Hz}, 1 \mathrm{H}, \mathrm{Ar}-\mathrm{H}), 6.71(\mathrm{~s}, 1 \mathrm{H}, \mathrm{NCH}), 6.73(\mathrm{~s}, 1 \mathrm{H}, \mathrm{NCH})$, $6.84(\mathrm{~s}, 1 \mathrm{H}, \mathrm{Ar}-\mathrm{H}), 7.17(\mathrm{~d}, J=8.46 \mathrm{~Hz}, 1 \mathrm{H}, \mathrm{Ar}-\mathrm{H}), 7.84(\mathrm{~s}, 1 \mathrm{H}, \mathrm{N}=\mathrm{CH})$. Anal. calcd. for $\mathrm{C}_{13} \mathrm{H}_{14} \mathrm{Cl}_{2} \mathrm{~F}_{6} \mathrm{~N}_{3} \mathrm{P}: \mathrm{C}, 36.47 ; \mathrm{H}, 3.30 ; \mathrm{N}, 9.81$. Found: C, 36.41; H, 3.04; N, 9.71.

1-[2-(4-Methoxybenzylideneamino)ethyl]-3-methyl-3H-imidazol-3-ium hexafluorophosphate (3c). Yellow solid (0.43 g, 36.82\%); mp 122-124 ${ }^{\circ} \mathrm{C}$. IR (KBr): $1644(\mathrm{C}=\mathrm{N}), 847 \mathrm{~cm}^{-1}$ $(\mathrm{P}-\mathrm{F}) .{ }^{1} \mathrm{H}$ NMR $\left(300 \mathrm{MHz}, \mathrm{DMSO}-d_{6}\right): \delta 3.01\left(\mathrm{~s}, 3 \mathrm{H}, \mathrm{CH}_{3}\right), 3.06\left(\mathrm{~s}, 3 \mathrm{H}, \mathrm{CH}_{3}\right), 3.14$ (t, J=5.16 $\left.\mathrm{Hz}, 2 \mathrm{H}, \mathrm{CH}_{2}\right), 3.70$ (t, J = 5.29 Hz, 2H, $\mathrm{CH}_{2}$ ), 6.13, 6.16 (AA', 2H, Ar-H), 6.70 (s, 1H, NCH), $6.79(\mathrm{~s}, 1 \mathrm{H}, \mathrm{NCH}), 6.83,6.86\left(\mathrm{BB}^{\prime}, 2 \mathrm{H}, \mathrm{Ar}-\mathrm{H}\right), 7.37(\mathrm{~s}, 1 \mathrm{H}, \mathrm{N}=\mathrm{CH})$. Anal. calcd. for $\mathrm{C}_{14} \mathrm{H}_{18} \mathrm{~F}_{6} \mathrm{~N}_{3} \mathrm{OP}: \mathrm{C}, 43.20 ; \mathrm{H}, 4.66 ; \mathrm{N}, 10.79$. Found: C, 42.29; H, 4.61; N, 10.55.

1-[2-(4-Nitrobenzylideneamino)ethyl]-3-methyl-3H-imidazol-3-ium hexafluorophosphate (3d). White solid (0.53 g, 43.70\%); mp 180-182 ${ }^{\circ} \mathrm{C}$. IR (KBr): $1641(\mathrm{C}=\mathrm{N}), 829 \mathrm{~cm}^{-1}(\mathrm{P}-\mathrm{F}) .{ }^{1} \mathrm{H}$ NMR (300 MHz, DMSO-d $\left.)_{6}\right): \delta .83\left(\mathrm{~s}, 3 \mathrm{H}, \mathrm{CH}_{3}\right), 4.01\left(\mathrm{t}, J=5.33 \mathrm{~Hz}, 2 \mathrm{H}, \mathrm{CH}_{2}\right), 4.52(\mathrm{t}, J=$ $\left.5.27 \mathrm{~Hz}, 2 \mathrm{H}, \mathrm{CH}_{2}\right), 7.67$ (s, 1H, NCH), 7.76 (s, 1H, NCH), 7.97, 7.99 (AA', 2H, Ar-H), 8.28, $8.31(\mathrm{BB}, 2 \mathrm{H}, \mathrm{Ar}-\mathrm{H}), 8.48(\mathrm{~s}, 1 \mathrm{H}, \mathrm{N}=\mathrm{CH}), 9.12(\mathrm{~s}, 1 \mathrm{H}, \mathrm{N}(\mathrm{H}) \mathrm{CN})$. Anal. calcd. for $\mathrm{C}_{13} \mathrm{H}_{15} \mathrm{~F}_{6} \mathrm{~N}_{4} \mathrm{O}_{2} \mathrm{P}: \mathrm{C}, 38.63 ; \mathrm{H}, 3.74 ; \mathrm{N}, 13.86$. Found: $\mathrm{C}, 38.48 ; \mathrm{H}, 3.58 ; \mathrm{N}, 13.62$.

1-[2-(3-Nitrobenzylideneamino)ethyl]-3-methyl-3H-imidazol-3-ium hexafluorophosphate (3e). White solid (0.65 g, 53.60\%); mp 133-135 ${ }^{\circ} \mathrm{C}$. IR (KBr): $1645(\mathrm{C}=\mathrm{N}), 838 \mathrm{~cm}^{-1}(\mathrm{P}-\mathrm{F}) .{ }^{1} \mathrm{H}$ NMR (300 MHz, DMSO-d $\left.)_{6}\right): \delta .09\left(\mathrm{~s}, 3 \mathrm{H}, \mathrm{CH}_{3}\right), 3.25\left(\mathrm{t}, J=5.20 \mathrm{~Hz}, 2 \mathrm{H}, \mathrm{CH}_{2}\right), 3.78(\mathrm{t}, J=$ $\left.5.32 \mathrm{~Hz}, 2 \mathrm{H}, \mathrm{CH}_{2}\right), 6.73(\mathrm{~s}, 1 \mathrm{H}, \mathrm{NCH}), 6.85$ (s, 1H, NCH), 6.88-7.62 (m, 4H, Ar-H), $7.78(\mathrm{~s}, 1 \mathrm{H}$, 
$\mathrm{N}=\mathrm{CH}$ ). Anal. calcd. for $\mathrm{C}_{13} \mathrm{H}_{15} \mathrm{~F}_{6} \mathrm{~N}_{4} \mathrm{O}_{2} \mathrm{P}: \mathrm{C}, 38.63 ; \mathrm{H}, 3.74 ; \mathrm{N}, 13.86$. Found: C, 38.05; H, 3.38;

N, 13.62 .

1-[2-(2-Hydroxybenzylideneamino)ethyl]-3-methyl-3H-imidazol-3-ium hexafluorophosphate (3f). Yellow solid (0.41 g, 36.42\%); mp 132-134 ${ }^{\circ} \mathrm{C}$. IR (KBr): $3422(\mathrm{O}-\mathrm{H}), 1640(\mathrm{C}=\mathrm{N}), 837$ $\mathrm{cm}^{-1}(\mathrm{P}-\mathrm{F}) .{ }^{1} \mathrm{H}$ NMR (300 MHz, DMSO-d $)$ ): $\delta 3.82\left(\mathrm{~s}, 3 \mathrm{H}, \mathrm{CH}_{3}\right), 3.99\left(\mathrm{t}, J=5.49 \mathrm{~Hz}, 2 \mathrm{H}, \mathrm{CH}_{2}\right)$, $4.52\left(\mathrm{t}, J=5.48 \mathrm{~Hz}, 2 \mathrm{H}, \mathrm{CH}_{2}\right), 6.85-7.42$ (m, 4H, Ar-H), 7.67 (s, 1H, NCH), 7.73 (s, 1H, NCH), $8.50(\mathrm{~s}, 1 \mathrm{H}, \mathrm{N}=\mathrm{CH}), 9.12(\mathrm{~s}, 1 \mathrm{H}, \mathrm{OH})$. Anal. calcd. for $\mathrm{C}_{13} \mathrm{H}_{16} \mathrm{~F}_{6} \mathrm{~N}_{3} \mathrm{OP}: \mathrm{C}, 41.61 ; \mathrm{H}, 4.29 ; \mathrm{N}$, 11.20. Found: C, 40.81; H, 4.19; N, 10.99.

1-[2-(4-Methylbenzylideneamino)ethyl]-3-methyl-3H-imidazol-3-ium hexafluorophosphate (3g). Yellow solid (0.38 g, 33.93\%); mp 131-133 ${ }^{\circ} \mathrm{C}$. IR (KBr): $1647(\mathrm{C}=\mathrm{N}), 833 \mathrm{~cm}^{-1}(\mathrm{P}-\mathrm{F}) .{ }^{1} \mathrm{H}$ NMR (300 MHz, DMSO-d $): \delta 1.55\left(\mathrm{~s}, 3 \mathrm{H}, \mathrm{CH}_{3}\right), 3.05\left(\mathrm{~s}, 3 \mathrm{H}, \mathrm{CH}_{3}\right), 3.16(\mathrm{t}, J=5.47 \mathrm{~Hz}, 2 \mathrm{H}$, $\left.\mathrm{CH}_{2}\right), 3.72\left(\mathrm{t}, J=5.47 \mathrm{~Hz}, 2 \mathrm{H}, \mathrm{CH}_{2}\right), 6.41(\mathrm{~s}, 1 \mathrm{H}, \mathrm{NCH}), 6.44(\mathrm{~s}, 1 \mathrm{H}, \mathrm{NCH}), 6.68-6.80(\mathrm{~m}, 4 \mathrm{H}$, Ar-H), 7.41 (s, $1 \mathrm{H}, \mathrm{N}=\mathrm{CH})$. Anal. calcd. for $\mathrm{C}_{14} \mathrm{H}_{18} \mathrm{~F}_{6} \mathrm{~N}_{3} \mathrm{P}: \mathrm{C}, 45.05 ; \mathrm{H}, 4.86 ; \mathrm{N}, 11.26$. Found: $\mathrm{C}$, 45.00; H, 5.36; N, 11.25 .

1-[2-(4-Bromobenzylideneamino)ethyl]-3-methyl-3H-imidazol-3-ium hexafluorophosphate (3h). White solid (0.39 g, 29.67\%); mp decomp. $>220{ }^{\circ} \mathrm{C}$. IR (KBr): $1641(\mathrm{C}=\mathrm{N}), 828 \mathrm{~cm}^{-1}(\mathrm{P}-$ F). ${ }^{1} \mathrm{H}$ NMR (300 MHz, DMSO-d $)$ ) $\delta 3.07\left(\mathrm{~s}, 3 \mathrm{H}, \mathrm{CH}_{3}\right), 3.18\left(\mathrm{t}, J=5.46 \mathrm{~Hz}, 2 \mathrm{H}, \mathrm{CH}_{2}\right), 3.73(\mathrm{t}$, $\left.J=5.46 \mathrm{~Hz}, 2 \mathrm{H}, \mathrm{CH}_{2}\right), 6.69(\mathrm{~s}, 1 \mathrm{H}, \mathrm{NCH}), 6.76(\mathrm{~s}, 1 \mathrm{H}, \mathrm{NCH}), 6.79-6.85$ (m, 4H, Ar-H), 7.45 (s, $1 \mathrm{H}, \mathrm{N}=\mathrm{CH}$ ). Anal. calcd. for $\mathrm{C}_{13} \mathrm{H}_{15} \mathrm{BrF}_{6} \mathrm{~N}_{3} \mathrm{P}: \mathrm{C}, 35.64 ; \mathrm{H}, 3.45 ; \mathrm{N}, 9.59$. Found: $\mathrm{C}, 35.17$; $\mathrm{H}$, $3.25 ; \mathrm{N}, 9.48$.

1-[2-(4-Hydroxy-3-methoxybenzylideneamino)ethyl]-3-methyl-3H-imidazol-3-ium hexafluorophosphate (3i). Yellow solid (0.52 g, 42.77\%); mp 178-180 ${ }^{\circ} \mathrm{C}$. IR (KBr): $1643(\mathrm{C}=\mathrm{N})$, $847 \mathrm{~cm}^{-1}(\mathrm{P}-\mathrm{F}) .{ }^{1} \mathrm{H}$ NMR $\left(300 \mathrm{MHz}, \mathrm{DMSO}-d_{6}\right): \delta 3.06\left(\mathrm{~s}, 3 \mathrm{H}, \mathrm{CH}_{3}\right), 3.07\left(\mathrm{~s}, 3 \mathrm{H}, \mathrm{CH}_{3}\right), 3.15(\mathrm{t}$, $\left.J=5.59 \mathrm{~Hz}, 2 \mathrm{H}, \mathrm{CH}_{2}\right), 3.71\left(\mathrm{t}, J=5.49 \mathrm{~Hz}, 2 \mathrm{H}, \mathrm{CH}_{2}\right), 5.98-6.31(\mathrm{~m}, 3 \mathrm{H}, \mathrm{Ar}-\mathrm{H}), 6.70(\mathrm{~s}, 1 \mathrm{H}$, $\mathrm{NCH}), 6.80(\mathrm{~s}, 1 \mathrm{H}, \mathrm{NCH}), 7.30(\mathrm{~s}, 1 \mathrm{H}, \mathrm{N}=\mathrm{CH})$. Anal. calcd. for $\mathrm{C}_{14} \mathrm{H}_{18} \mathrm{~F}_{6} \mathrm{~N}_{3} \mathrm{O}_{2} \mathrm{P}: \mathrm{C}, 41.49 ; \mathrm{H}$, 4.48; N, 10.37. Found: C, 40.81; H, 4.47; N, 10.20.

\section{Acknowledgements}

We are grateful to the National Natural Science Foundation of China (No. 20672046) and the Guangdong Natural Science Foundation (No. 8151063201000016) for financial support.

\section{References}

1. Tseng, M. C.; Kan, H. C.; Chu, Y. H. Tetrahedron Lett. 2007, 48, 9085.

2. Ranu, B. C.; Jana, R.; Sowmiah, S. J. Org. Chem. 2007, 72, 3152.

3. Galinski, M.; Lewandowski, A.; Stepniak, I. Electrochim. Acta 2006, 51, 5567.

4. Ganeshpure, P. A.; George, G.; Das, J. Arkivoc 2007, (viii), 273. 
5. Wasserscheid, P.; Keim, W. Angew Chem. Int. Ed. 2000, 39, 3772.

6. Singh, B.; Sekhon, S. S. Chem. Phys. Lett. 2005, 414, 34.

7. Noda, A.; Watanabe, M. Electrochim. Acta 2000, 45, 1265.

8. Lewandowski, A.; Swiderska, A. Solid State Ionics 2004, 169, 21.

9. Li, X.; Zhao, D.; Fei, Z.; Wang, L. Sci China Ser B: Chem. 2006, 49, 385.

10. Jain, N.; Kumar, A.; Chauhan, S.; Chauhan, S. M. S. Tetrahedron 2005, 61, 1015.

11. Gayet, F.; Marty, J. D.; Viguerie, N. L. Arkivoc 2008, (xvii), 61.

12. Santos, J. E.; Edward, R. D. Eder; Cavalheiro, T. G. Carbohydr. Polym. 2005, 60, 277.

13. Balasubramanian, K. P.; Parameswari, K.; Chinnusamy, V.; Prabhakaran, R.; Natarajan, K. Spectrochim. Acta A 2006, 65, 678.

14. Karvembu, R.; Hemalatha, S.; Prabhakaran, R.; Natarajan, K. Inorg. Chem. Commun. 2003, $6,486$.

15. Chandra, S.; Gupta, K. Trans Metal Chem. 2002, 27, 196.

16. Balsells, J.; Mejorado, L.; Phillips, M.; Ortega, F.; Aguirre G.; Somanathan R.; Walsh, P. J. Tetrahedron: Asymm. 1998, 9, 4135.

17. Rihter, B.; Srittari, S.; Hunter, S.; Masnovi J. J. Am. Chem. Soc. 1993, 115, 3918.

18. Occhipinti, G.; Jensen, V. R.; Bjrsvik, H. R. J. Org. Chem. 2007, 72, 3561.

19. Satyanarayana, V. S. V.; Sreevani, P.; Sivakumar, A.; Vijayakumar, V. Arkivoc 2008, (xvii), 221.

20. Wang, H. Q.; Zhou, W. P.; Liu, Z. J. Chinese J. Struct. Chem. 2006, 25, 1105.

21. Peng, Y.; Song, G. Catal. Commun. 2007, 8, 111.

22. Sheldrick, G. M. SADABS, University of Göttingen, Germany 1996.

23. Sheldrick, G. M. SHELXL-97, University of Göttingen, Germany 1997. 